\title{
OBTENÇÃO DE PROPRIEDADES TERMODINÂMICAS DE HIDROCARBONETOS NA REGIÃO CRÍTICA UTILIZANDO O MÉTODO DE MONTE CARLO
}

\author{
M.H. FERNANDES ${ }^{1}$, L. MAURIERI ${ }^{1}$, M. L. L. PAREDES ${ }^{1}$ e L. BATISTA ${ }^{2}$ \\ ${ }^{1}$ Universidade do Estado do Rio de Janeiro, Faculdade de Tecnologia. \\ ${ }^{2}$ Universidade do Estado do Rio de Janeiro, Instituto de Química. \\ E-mail para hnrq.fernandes@gmail.com
}

\begin{abstract}
RESUMO - O presente trabalho investigou como o método de Monte Carlo pode ser utilizado para prever o ponto crítico do propano. Foram calculados os coeficientes de expansividade térmica e compressibilidade isotérmica em diferentes condições de temperatura e pressão no ensemble isobárico-isotérmico, de modo que a divergência dessas propriedades aponte a região do ponto crítico. De acordo com os resultados e condições avaliadas o método prevê região do ponto crítico acima do valor experimental.
\end{abstract}

\section{INTRODUÇÃO}

Derivados do petróleo estão em nosso cotidiano desde simples embalagens até materiais para construção de veículos, eletrodomésticos ou mesmo próteses compatíveis com o organismo humano. De modo a atender à grande demanda por derivados de petróleo, a produção nacional chegou a atingir 2,73 milhões de barris por dia em dezembro do ano passado, o que significou um novo recorde em termos de produção de petróleo (ANP, 2017). Tendo em vista a redução dos principais custos de produção, fazem-se então necessários estudos de mapeamento e caracterização do óleo bruto em variadas condições, de modo que seja possível otimizar etapas de produção e distribuição de derivados de petróleo.

Normalmente, a aquisição de propriedades termodinâmicas é feita por meio de equações de estado, estas costumam apresentar baixas exatidões em regiões próximas ao ponto crítico (De Hemptinne et al, 1998). Outra maneira de obtenção de dados são ensaios experimentais que, por sua vez, podem necessitar de aparato instrumental complexo e de elevado custo. Uma alternativa, relativamente recente, para obtenção de propriedades físicoquímicas, mas de pouco uso na indústria, é a utilização de simulações moleculares. Essa metodologia pode ser de grande importância para obtenção de propriedades de interesse da indústria petroquímica em situações onde os modelos determinísticos convencionais não apresentam respostas satisfatórias ou experimentos são enviáveis.

Dentre as propriedades que podem ser obtidas através de simulação molecular, destacam-se o coeficiente de expansividade térmica e o coeficiente de compressibilidade isotérmica. A análise das flutuações, além da divergência destes parâmetros na temperatura e 
pressão críticas (Tc e Pc, respectivamente) pode indicar o limite de estabilidade de fases e, por conseguinte, serem utilizadas no mapeamento da região crítica.

O método usual de inferência do ponto crítico líquido-vapor por simulação molecular é através da extrapolação dos resultados de equilíbrio até o ponto crítico. Nesse trabalho, está sendo empregada uma técnica alternativa, na qual mapeiam-se as condições de temperatura e pressão nas quais algumas propriedades "divergem" no ponto crítico, ou seja, tendem a valores infinitos nesse ponto. Isso vale para expansividade térmica, compressibilidade isotérmica e capacidade calorífica.

\section{METODOLOGIA}

O coeficiente de expansividade térmica e coeficiente de compressibilidade isotérmica ( $\alpha_{\mathrm{P}}$ e $\beta_{\mathrm{T}}$, respectivamente) foram calculadas por meio do Método de Monte Carlo no ensemble isobárico-canônico (NPT) e campo de força TraPPE-UA. Avaliou-se a estabilização da energia, densidade e pressão do virial do sistema ao longo da simulação, que foi feita em um número de etapas suficientemente grande para garantir um conjunto de flutuações dessas propriedades desprezível. Foram realizadas simulações de 50 mil ciclos de Monte Carlo na etapa de termalização, blocados a cada 10 mill ciclos, e simulações de 50 mil ciclos de Monte Carlo na etapa de tomada de dados (equilíbrio).

Para mapeamento da temperatura crítica, estudaram-se sistemas contendo 200 e 2000 moléculas de propano, variando a temperatura na isóbara crítica experimental (42,5 bar) em passos de $5 \mathrm{~K}$ de $350 \mathrm{~K}$ a $390 \mathrm{~K}$ para o sistema de 200 moléculas e $354,9 \mathrm{~K}$ a $384,9 \mathrm{~K}$ para o sistema contendo 2000 moléculas. De forma semelhante, a região da pressão crítica foi analisada fixando a temperatura da simulação na respectiva temperatura crítica experimental do propano $(369,9 \mathrm{~K})$ e variando a pressão em passos de 5 bar entre 30 bar e 60 bar para o sistema de 200 moléculas e em passos de 10 bar de 12,5 bar a 72,5 bar para sistemas com 2000 moléculas. Se temperatura e pressão críticas relativas ao campo de força escolhido foram iguais às experimentais, haverá divergência de $\alpha_{\mathrm{P}}$ e $\beta_{\mathrm{T}}$ no ponto crítico. Caso a simulação seja realizada em temperatura ou pressão inferior às críticas relativas ao campo de força, as simulações poderão ocorrer em sistemas monofásicos apenas líquidos, apenas vapor, ou poderá ser encontrada uma descontinuidade relativa à transição líquido-vapor.

Todas as simulações foram conduzidas em um dos principais programas utilizados para simulações de Monte Carlo, o MCCCS Towhee (Martin, 2017). Entretanto, como esse programa em sua versão original não reporta os resultados para os coeficientes de interesse nesse trabalho, foi gerada na UERJ uma versão do programa capaz de calcular tais propriedades através dos cálculos de flutuações em volume e energia no ensemble. Tais modificações foram validadas contra dados experimentais das propriedades calculadas para metano e $\mathrm{CO} 2$, com excelente aderência entre resultados de simulação e experimentais. Para fins comparativos, os resultados obtidos com simulação molecular foram comparados com uma equação de estado multiparamétrica, GERG-2008 (Kunz e Wagner, 2012).

\section{RESULTADOS}




\subsection{Análise das Flutuações na Termalização e no Equilíbrio}

De acordo com a Figura 1, logo a partir do segundo bloco de ciclos Monte Carlo da etapa de termalização, já se alcançam valores para flutuação de $\alpha_{\mathrm{P}}$ e $\beta_{\mathrm{T}}$ compatíveis com os valores obtidos na etapa de equilíbrio. A Figura 1 indica que em 20 mil ciclos Monte Carlo o sistema já se encontra termalizado. Para enfatizar a extensão das simulações realizadas, os valores médios calculados, desconsiderando os valores dos primeiros 10 mil cilos, para $\alpha_{P} \mathrm{e}$ $\beta_{\mathrm{T}}$ na etapa de termalização são $1,55 \times 10^{-3} \mathrm{~K}^{-1}$ e $3,65 \times 10^{-4} \mathrm{MPa}^{-1}$ enquanto que no equilíbrio, obtêm-se os valores médios de $1,53 \times 10^{-3} \mathrm{~K}^{-1}$ e $3,52 \times 10^{-4} \mathrm{Mpa}^{-1}$, respectivamente. Logo, as simulações foram realizadas com o número de ciclos suficientes para caracterização da região de ponto crítico.

Figura 1 - Flutuações de $\alpha_{\mathrm{P}}$ e $\beta_{\mathrm{T}}$ x bloco para sistema com 2000 moléculas a 42,5 bar e 369,9 K usando TraPPE-UA.

(a)

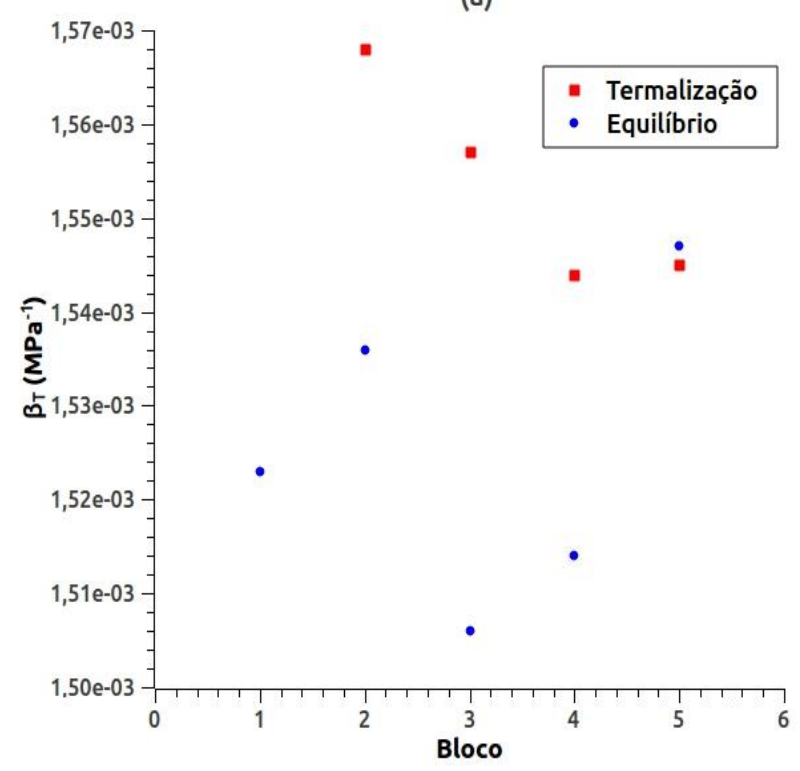

(b)

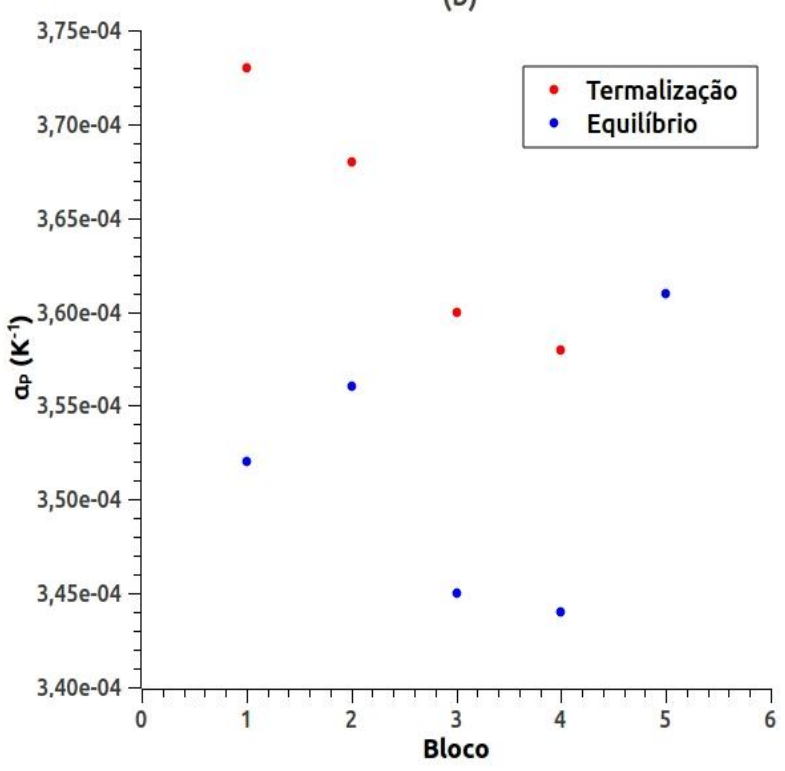

\subsection{Mapeamento da Pressão Crítica}

No mapeamento da pressão crítica, foram observados diferentes resultados de acordo com o tamanho do sistema estudado. Os resultados estão apresentados na Figura 2, onde os resultados obtidos com o campo de força TraPPE-UA são comparados com os resultados da GERG-2008. De acordo com a Figura 2, os resultados da simulação molecular para sistemas com 200 moléculas (Fig. 2-a) não indicaram mudança de fase, onde apenas valores de $\alpha_{\mathrm{P}} \mathrm{e} \beta_{\mathrm{T}}$ típicos de líquido foram encontrados. Já a simulação molecular com 2000 moléculas (Fig. 2b) conseguiu verificar uma nítida transição de fases ocorrendo no intervalo de 32,5 bar e 42,5 bar. Valores gerados pela GERG 2008 (Fig. 2-c) apresentam claramente a divergência para as propriedades estudadas em 42,5 bar, indicando o resultado esperado com a técnica empregada nesse trabalho, caso a temperatura ou pressão mantida constante seja igual à crítica. 
Figura 2. Variação de $\alpha_{P}$ e $\beta_{\text {T }}$ contra Pressão a 369,9 K. (a) Simulação Molecular usando TraPPE-UA com 200 moléculas. (b) Simulação Molecular usando TraPPE-UA com 2000 moléculas. (c) GERG 2008.

(a)

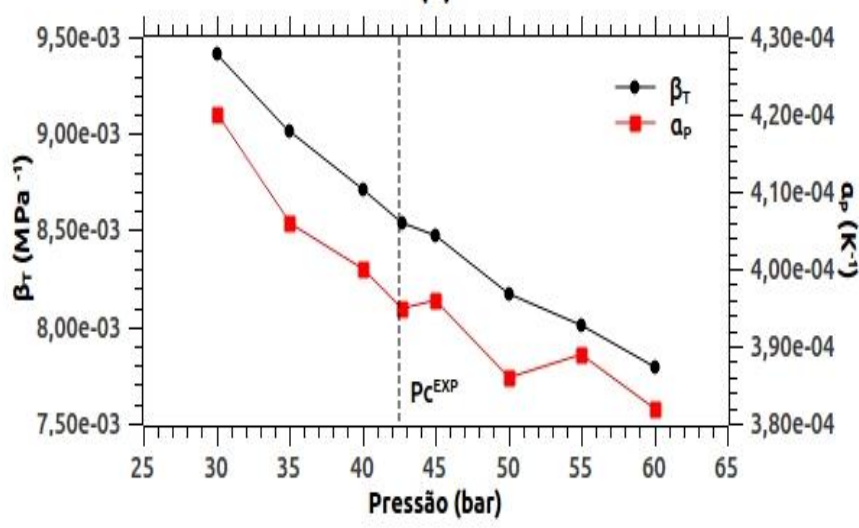

(c)

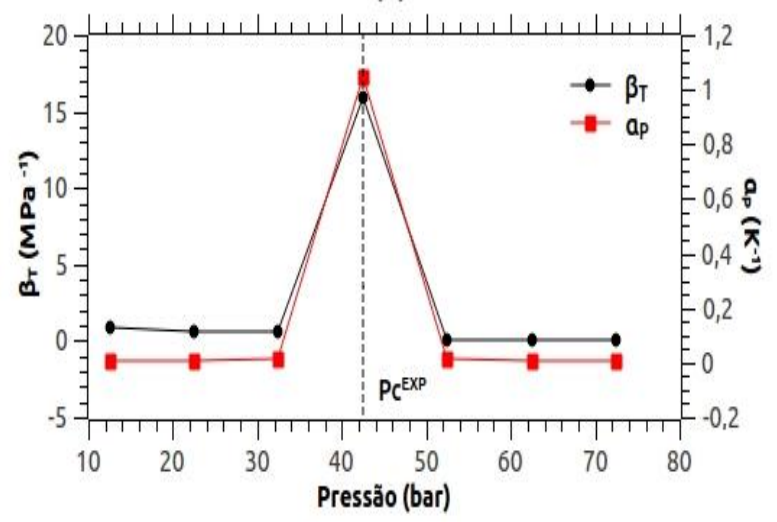

(b)

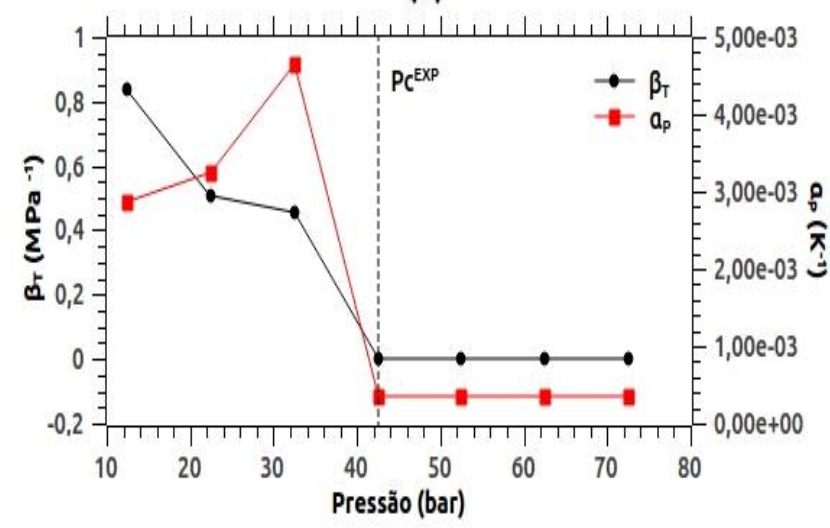

\subsection{Mapeamento da Temperatura Crítica}

Não foram observadas transições de fase para simulação molecular no mapeamento da temperatura crítica, independente do tamanho do sistema considerado, sejam 200 ou 2000 moléculas de propano (Fig. 3 - a e b). A análise crítica dos dados indica que no intervalo de temperatura investigado o sistema se encontra em fase líquida. Novamente, as propriedades calculadas com a GERG-2008 apresentaram a esperada divergência no valor de 369,9 K. Apesar de simulações extensas, de modo que o sistema tenha liberdade para apresentar flutuações em suas propriedades, e a inclusão de um grande número de moléculas na caixa de simulação, o método de Monte Carlo com o campo de força utilizado não previu corretamente os parâmetros críticos do propano.

Vê-se na Figura 2-b que a pressão de vapor para temperatura igual a 369,9 K se encontra entre 32,5 e 42,5 bar. Como o mapeamento de Tc foi feito fixando-se o valor da 
pressão em 42,5 bar e não foi observada nenhuma transição de fase no intervalo de 355 a 385 $\mathrm{K}$, assume-se que o campo de força estudado leva a uma temperatura crítica acima de $385 \mathrm{~K}$.

Figura 3. Variação de $\alpha_{\mathrm{P}}$ e $\beta_{\mathrm{T}}$ contra Temperatura a 42,5 bar. (a) Simulação Molecular usando TraPPE-UA com 200 moléculas. (b) Simulação Molecular usando TraPPE-UA com 2000 moléculas. (c) GERG 2008.

(a)

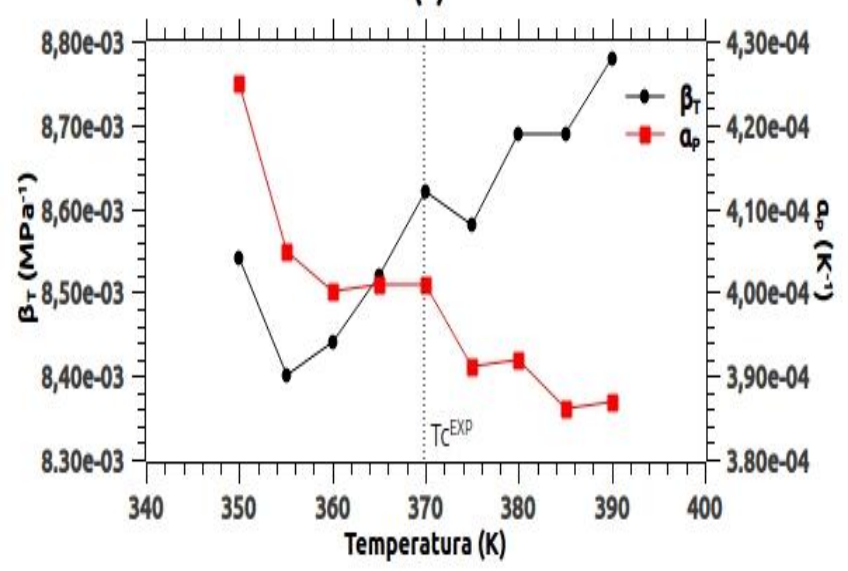

(c)

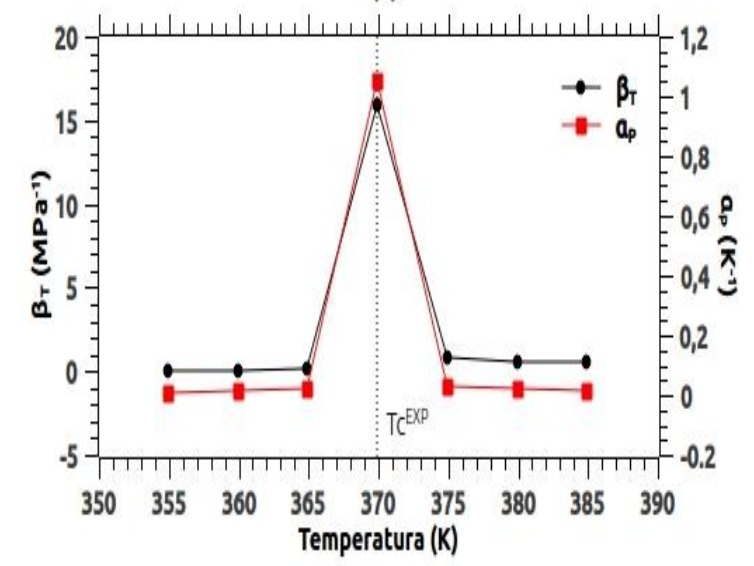

(b)

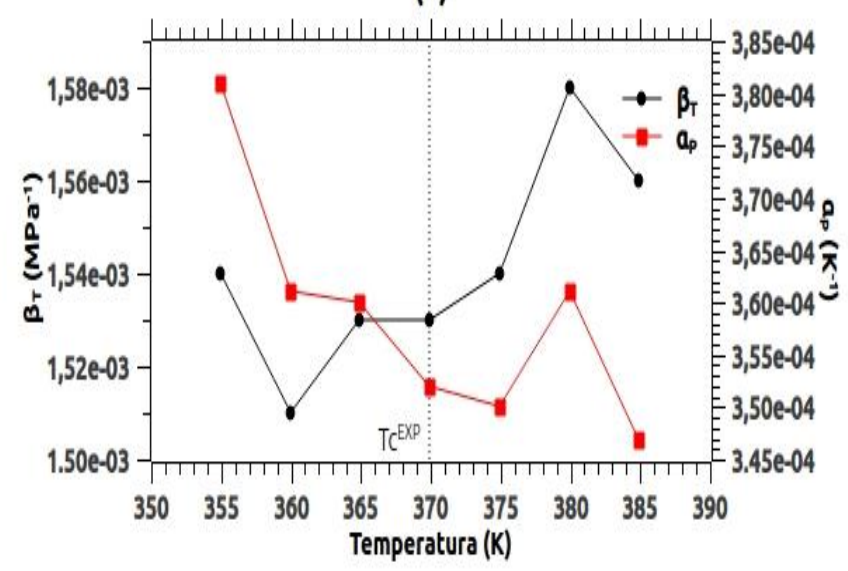

\section{CONCLUSÃO}

O presente estudo apresenta a simulação molecular pelo método de Monte Carlo como uma alternativa para obtenção de propriedades termodinâmicas de sistemas de interesse da indústria do petróleo. No presente caso, foi avaliado como a simulação de Monte Carlo pode ser utilizada para mapear a região de ponto crítico do propano. Foi avaliada como as propriedades são afetadas pelo tamanho de caixa, sendo verificado que um número reduzido de moléculas (200) não pode ser utilizado para representação do sistema, visto que no intervalo de pressões estudado não foi verificado a transição de fase esperada para o propano.

A simulação molecular para o ensemble NPT, usando os campos de força do TraPPEUA indica uma temperatura crítica acima da experimental, apesar de não alcançado seu valor 
no presente estudo. Mais estudos se fazem necessários para melhor utilizar-se o método de Monte Carlo no ensemble NPT para predições da região de ponto crítico.

\section{NOMENCLATURA}

ap. Coeficiente de expansividade térmica $\left(\mathrm{K}^{-1}\right)$.

$\beta_{\text {T. }}$ Coeficiente isotérmico de compressibilidade $\left(\mathrm{MPa}^{-1}\right)$.

MCCCS Towhee. Monte Carlo for Complex Chemical Systems.

T. Temperatura $(\mathrm{K})$.

Tc. Temperatura Crítica (K).

P. Pressão (bar).

Pc. Pressão Crítica (bar).

\section{REFERÊNCIAS}

ANP. Boletim informativo de Dezembro de 2016. Bol. inf. da Agência Nacional do Petróleo Gás Natural e Combustível, pág. 4, 2017.

HEMPTINNE, J.-C. de; BARREAU, A.; UNGERER, P.; BEHAR, E. Evaluation of equations of state at high pressure for light hydrocarbons. Thermodynamic Modeling and Materials Data Engineering, v. 1, págs. 53-70, 1998.

KUNZ, O.; WAGNER, W. The GERG-2008 wide-range equation of state for natural gases and other mixtures: an expansion of GERG-2004. J. Chem. Eng. Data, v. 57, págs. 3032-3091, 2012.

MARTIN, M. http://towhee.sourceforge.net/, recuperado em 16 de Março de 2017.

MARTIN, M. G.; SIEPMANN, J. I. Transferable potentials for phase equilibria. 1. unitedatom description of n-alkanes. J. Phys. Chem. B, v. 102, págs. 2569-2577, 1998. 